\title{
Cosmic dissonance: are new physics or systematics behind a short sound horizon?
}

\author{
Nikki Arendse ${ }^{1}$, Radosław J. Wojtak ${ }^{1}$, Adriano Agnello ${ }^{1}$, Geoff C.-F. Chen ${ }^{2}$, Christopher D. Fassnacht ${ }^{2}$, \\ Dominique Sluse ${ }^{3}$, Stefan Hilbert ${ }^{4,5}$, Martin Millon ${ }^{6}$, Vivien Bonvin ${ }^{6}$, Kenneth C. Wong ${ }^{7}$, Frédéric Courbin ${ }^{6}$, \\ Sherry H. Suyu ${ }^{8,9,10}$, Simon Birrer ${ }^{11,12}$, Tommaso Treu ${ }^{11}$, and Leon V. E. Koopmans ${ }^{13}$ \\ 1 DARK, Niels-Bohr Institute, Lyngbyvej 2, 2100 Copenhagen, Denmark \\ e-mail: nikki.arendse@nbi.ku.dk \\ 2 Physics Department UC Davis, 1 Shields Ave., Davis, CA 95616, USA \\ 3 STAR Institute, Quartier Agora - Allée du six Août, 19c B-4000 Liège, Belgium \\ ${ }^{4}$ Exzellenzcluster Universe, Boltzmannstr. 2, 85748 Garching, Germany \\ 5 Ludwig-Maximilians-Universität, Universitäts-Sternwarte, Scheinerstr. 1, 81679 München, Germany \\ ${ }^{6}$ Laboratoire d'Astrophysique, École Politechnique Fédérale de Lausanne (EPFL), Obs. de Sauverny, 1290 Versoix, Switzerland \\ 7 Kavli IPMU (WPI), UTIAS, The University of Tokyo, Kashiwa, Chiba 277-8583, Japan \\ 8 Max-Planck-Institut für Astrophysik, Karl-Schwarzschild-Str. 1, 85748 Garching, Germany \\ 9 Physik-Department, Technische Universität München, James-Franck-Straße 1, 85748 Garching, Germany \\ 10 Academia Sinica Institute of Astronomy and Astrophysics (ASIAA), 11F of ASMAB, No.1, Sect. 4, Roosevelt Rd, Taipei 10617, \\ Taiwan \\ 11 Department of Physics and Astronomy, University of California, Los Angeles, CA 90095, USA \\ 12 Kavli Institute for Particle Astrophysics and Cosmology and Department of Physics, Stanford University, Stanford, CA 94305 , \\ USA \\ 13 Kapteyn Astronomical Institute, University of Groningen, PO Box 800, 9700 AV Groningen, The Netherlands
}

Received 17 September 2019 / Accepted 7 May 2020

\begin{abstract}
Context. Persistent tension between low-redshift observations and the cosmic microwave background radiation (CMB), in terms of two fundamental distance scales set by the sound horizon $r_{\mathrm{d}}$ and the Hubble constant $H_{0}$, suggests new physics beyond the Standard Model, departures from concordance cosmology, or residual systematics.

Aims. The role of different probe combinations must be assessed, as well as of different physical models that can alter the expansion history of the Universe and the inferred cosmological parameters.

Methods. We examined recently updated distance calibrations from Cepheids, gravitational lensing time-delay observations, and the tip of the red giant branch. Calibrating the baryon acoustic oscillations and type Ia supernovae with combinations of the distance indicators, we obtained a joint and self-consistent measurement of $H_{0}$ and $r_{\mathrm{d}}$ at low redshift, independent of cosmological models and $\mathrm{CMB}$ inference. In an attempt to alleviate the tension between late-time and CMB-based measurements, we considered four extensions of the standard $\Lambda \mathrm{CDM}$ model.

Results. The sound horizon from our different measurements is $r_{\mathrm{d}}=\left(137 \pm 3^{\text {stat. }} \pm 2^{\text {syst. }}\right) \mathrm{Mpc}$ based on absolute distance calibration from gravitational lensing and the cosmic distance ladder. Depending on the adopted distance indicators, the combined tension in $H_{0}$ and $r_{\mathrm{d}}$ ranges between 2.3 and $5.1 \sigma$, and it is independent of changes to the low-redshift expansion history. We find that modifications of $\Lambda \mathrm{CDM}$ that change the physics after recombination fail to provide a solution to the problem, for the reason that they only resolve the tension in $H_{0}$, while the tension in $r_{\mathrm{d}}$ remains unchanged. Pre-recombination extensions (with early dark energy or the effective number of neutrinos $N_{\text {eff }}=3.24 \pm 0.16$ ) are allowed by the data, unless the calibration from Cepheids is included.

Conclusions. Results from time-delay lenses are consistent with those from distance-ladder calibrations and point to a discrepancy between absolute distance scales measured from the CMB (assuming the standard cosmological model) and late-time observations. New proposals to resolve this tension should be examined with respect to reconciling not only the Hubble constant but also the sound horizon derived from the $\mathrm{CMB}$ and other cosmological probes.
\end{abstract}

Key words. gravitational lensing: strong - cosmological parameters - distance scale - early Universe

\section{Introduction}

At the onset of matter-radiation decoupling after the Big Bang, photon-baryon fluid underwent oscillations whose characteristic physical scale is described by the so-called sound horizon, $r_{\mathrm{s}}$. This leaves a characteristic imprint on large-scale distribution of baryons, with its characteristic size fixed in the comoving coordinates and equal to the sound horizon at the drag epoch, $z_{\mathrm{d}}$, given by

$r_{\mathrm{d}} \equiv r_{\mathrm{s}}\left(z_{\mathrm{d}}\right)=\int_{z_{\mathrm{d}}}^{\infty} \frac{c_{\mathrm{s}} \mathrm{d} z}{H(z)}$

where $c_{\mathrm{s}}$ is the sound speed in the primordial plasma, and $H(z)$ is the Hubble parameter.

The sound horizon $r_{\mathrm{d}}$ is robustly determined from the cosmic microwave background measurements (CMB), if the 
Standard Model of particle physics as well as the standard cosmological model in the pre-recombination Universe are adopted (Planck Collaboration VI 2020). Alternatively, it can be measured at later times, from the baryon acoustic oscillation (BAO) peak in the two-point spatial correlation function of galaxies and quasars. The latter is an angular measurement, which can be converted into a physical $r_{\mathrm{d}}$ measurement through independent distance calibrations (see e.g. Heavens et al. 2014; Bernal et al. 2016; Verde et al. 2017; Arendse et al. 2019; Aylor et al. 2019). The parameter $r_{\mathrm{d}}$ is intimately linked to the current expansion rate of the Universe, the Hubble constant $H_{0}$, since BAO measurements constrain the product of $H_{0}$ and $r_{\mathrm{d}}$.

Accurate distance measurements from CMB-independent observations can be used to determine $r_{\mathrm{d}}$ and $H_{0}$ in a way that is truly independent of early-Universe physics. Therefore, these measurements can test our understanding of the concordance cosmology and the Standard Model of particle physics, through low-redshift measurements only. Type Ia supernovae, calibrated by Cepheids with three independent distance anchors (parallaxes in the Milky Way, detached eclipsing binaries in the LMC and maser galaxy NGC 4258), provide the most precise distance calibration to date, as performed by the Supernovae and $\mathrm{H}_{0}$ for the Equation of State of dark energy project (SHOES; Riess et al. 2019). Another powerful way of obtaining absolute distances is by using strongly lensed quasar systems, which extend to higher redshifts than the Cepheids. The $\mathrm{H}_{0}$ Lenses in COSMOGRAIL's Wellspring collaboration (HOLiCOW, Suyu et al. 2017) has provided few-percent-level precision constraints on $H_{0}$ from timedelay cosmology. Over the whole sample, the effect of known systematics is at a $\lesssim 1 \%$ level, which is currently negligible with respect to the statistical uncertainties (Millon et al. 2020). The latest results from SHOES and HOLiCOW indicate a strong tension in the Hubble constant $H_{0}$ between late-time observations (CMB-independent probes including primarily type Ia $\mathrm{SNe}$, lensing and $\mathrm{BAO}$ ) and $\mathrm{CMB}$-based measurements, within a flat $\Lambda C D M$ model. Previous results based on four lenses alone (Arendse et al. 2019; Taubenberger et al. 2019) resulted in a $2 \sigma$ discrepancy, while a six-lens analysis (Wong et al. 2020) gave a $3 \sigma$ tension. When combined with the distance-ladder results from SHOES, the tension increases to a $5 \sigma$ level, still adopting a flat $\Lambda C D M$ cosmological model. It is worth noting that the tension between the late-time and CMB-based measurements of $H_{0}$ is mildly lowered by the recent measurement making use of precise distance calibration from the tip of the red giant branch (TRGB), as measured by the Carnegie-Chicago Hubble Project (herafter CCHP, Freedman et al. 2019). These measurements fall between those from SH0ES and the CMB, at $1.7 \sigma$ and $1.2 \sigma$ differences, respectively. For the sake of completeness, it is also worth mentioning that the Planck value of the Hubble constant is recovered in a CMB-independent but model-dependent analysis of BAO observations with the prior on the baryon density from the standard Big-Bang nucleosynthesis (Cuceu et al. 2019; Addison et al. 2018).

In this work, we revisit the claimed tension between late-time observations and the $\mathrm{CMB}$ in terms of the sound horizon and the Hubble constant, by making use of recent updated distance calibrations from gravitational time-delay lenses (HOLiCOW), Cepheids (SHOES), and TRGB (CCHP). Through our methods (summarised in Sect. 2.2), we obtain measurements of $r_{\mathrm{d}}$ for different combinations of late-time distance calibrations in a manner that is almost completely independent of any cosmological model. Moreover, we investigate selected extensions to the standard $\Lambda$ CDM model that were recently proposed as possible solutions to the Hubble tension. Such new models attempt to reconcile the tension by modifying the expansion history of the Standard Model either before or after recombination, hereafter early-time and late-time modifications, and thus increasing the Hubble constant derived from the CMB. We demonstrate that the late-time extensions fail to provide a solution to the problem, for the reason that they only succeed in alleviating the tension in $H_{0}$, while the tension in $r_{\mathrm{d}}$ remains unchanged. Our analysis emphasises the importance of comparing at least $H_{0}$ and $r_{\mathrm{d}}$ derived from late-time observations and the $\mathrm{CMB}$ when testing new models devised to mitigate the Hubble constant tension.

This paper is structured as follows. Section 2 describes the late-time measurements of $r_{\mathrm{d}}$ and $H_{0}$, including the different data sets, models and inference methods that are used. In Sect. 3, we outline how the late-time measurements are compared with CMB inference and extensions of the concordance scenario. Our results are described in Sect. 4, and our conclusions feature in Sect. 5.

\section{Late-time measurements: data and methods}

The values of $r_{\mathrm{d}}$ and $H_{0}$ can be constrained by employing several CMB-independent probes at $0<z<2$, in this paper referred to as late-time measurements. In Sect. 2.1, we provide an overview of the data sets that we use in our analysis. Section 2.2 introduces the models we chose to fit the Hubble diagram and interpolate up to redshift zero. By choosing models that are independent of cosmology, we minimise the systematic uncertainty associated with cosmological model choices. Details about the inference are discussed in Sect. 2.3, and functional tests are shown in Appendix B.

\subsection{Data sets}

The shape of the late-time expansion of the Universe has been mapped precisely with type Ia supernovae ( $\mathrm{SNe}$ ). In this work, we used relative distance moduli from the Pantheon sample (Scolnic et al. 2018).

Information about $r_{\mathrm{d}}$ was introduced by adding BAO measurements, which constrain the product of $H_{0}$ and $r_{\mathrm{d}}$. Our main results are obtained for the Hubble parameters $H(z)$ and the transverse comoving distances $D_{\mathrm{M}}(z)$ determined from the Baryon Oscillation Spectroscopic Survey (BOSS; Alam et al. 2017). Additionally, we looked into the effect of adding BAO constraints from the correlation of $\operatorname{Ly} \alpha$ forest absorption and quasars in the extended Baryon Oscillation Spectroscopic Survey (eBOSS; de Sainte Agathe et al. 2019; Blomqvist et al. 2019) and several isotropic BAO measurements. The isotropic measurements do not contain sufficient statistics to measure $H(z)$ and $D_{\mathrm{M}}(z)$ separately, but combine them in the volume-averaged distance $D_{\mathrm{V}}=\left(c z D_{\mathrm{M}}^{2}(z) H^{-1}(z)\right)^{1 / 3}$. We included two measurements from the reconstructed six-degree Field Galaxy Survey (Carter et al. 2018), two from eBOSS by Bautista et al. (2018), Ata et al. (2018), and three from the WiggleZ Dark Energy Survey (Kazin et al. 2014).

Both $\mathrm{SNe}$ and $\mathrm{BAO}$ measurements provide only relative distances, thus their distance scale needs to be calibrated with absolute distance measurements. Time-delay and angular-diameter distances to strongly lensed quasars, obtained by the HOLiCOW collaboration, provide such an absolute calibration of cosmological distances (see e.g. Suyu et al. 2017, and references therein). Results from a fifth and a sixth lensed quasar system were recently obtained (Chen et al. 2019; Rusu et al. 2019; Bonvin et al. 2019; Sluse et al. 2019), including new distance 
measurements on previous lensed quasar systems using new data and analyses (Chen et al. 2019; Jee et al. 2019). In this work, we used complete constraints on distances from observations of the six lensed quasar systems, as summarised in Wong et al. (2020). The information from the lensed quasars has been modelled self consistently, together with the relative distance indicators ( $\mathrm{SNe}$, BAO).

Keeping the lensing data as our primary calibration of the absolute distance scale in all fits, we also included two optional priors given by recent local determinations of the Hubble constant. The first is the latest SHOES measurement yielding $H_{0}=$ $74.03 \pm 1.42 \mathrm{~km} \mathrm{~s}^{-1} \mathrm{Mpc}^{-1}$ (Riess et al. 2019). The second is based on calibrating distances with the tip of the red giant branch (TRGB), a standard candle alternative to Cepheids. Here, analyses carried out by two separate groups have resulted in different values for $H_{0}$ : Yuan et al. (2019) found $72.4 \pm 2.0 \mathrm{~km} \mathrm{~s}^{-1} \mathrm{Mpc}^{-1}$, while CCHP obtained $69.6 \pm 2.0 \mathrm{~km} \mathrm{~s}^{-1} \mathrm{Mpc}^{-1}$ (Freedman et al. 2019, 2020). In order to include both the highest and lowest latetime measurements of $H_{0}$, we chose to use the CCHP results for the TRGB and SHOES results for Cepheids in our analysis. Since there is a partial overlap in the galaxy samples considered for the TRGB and Cepheid measurements, the two calibrations have only been applied separately.

Finally, quasars were optionally used as secondary standard candles at high redshifts, by means of a relation between their UV and X-ray luminosities (Risaliti \& Lusso 2019). We did this in one of our inference runs in Table 4, as an independent check.

Our constraints on the late-time expansion are largely based on data sets and models that we explored in a previous work (Arendse et al. 2019). The difference with previous data sets is the inclusion of two additional quasar-lens measurements (Chen et al. 2019; Rusu et al. 2019), Ly $\alpha$ BAO measurements at $z=2.34$ and 2.35, several volume-averaged BAO measurements $\left(D_{\mathrm{V}} \mathrm{BAO}\right)$, and the combination with the Cepheid distance ladder or the TRGB calibration.

\subsection{Models}

Measuring $r_{\mathrm{d}}$ and $H_{0}$ from the observations described above requires adopting a model of the expansion history. This is usually done by means of employing the standard $\Lambda \mathrm{CDM}$ model, but any tension among different $r_{\mathrm{d}}$ and $H_{0}$ measurements in the $\Lambda C D M$ framework may mean that the $\Lambda$ CDM expansion history is not necessarily an adequate model choice. Instead of employing different extensions to $\Lambda \mathrm{CDM}$ to overcome this issue, we used three different models of polynomial parametrisations, which are completely agnostic about the underlying expansion history. This allows us to make an inference of $r_{\mathrm{d}}$ and $H_{0}$ that is based solely on observational data, and that does not rely on cosmology.

The specifications of the three polynomial parametrisations (hereafter referred to as model 1,2 and 3) are listed in Table 1. Model 1 adopts a polynomial expansion of $H(z)$ (Weinberg 1972; Visser 2004), model 2 expands the luminosity distance $D_{\mathrm{L}}{ }^{1}$ as a polynomial in $\log (1+z)$ (Risaliti \& Lusso 2019), and model 3 describes transverse comoving distances $D_{\mathrm{M}}$ by polynomials in $z /(1+z)$ (Cattoën \& Visser 2007; Li et al. 2020). For model 1 , comoving distances were obtained from $H(z)$ through direct numerical integration of

$d_{\mathrm{c}}(z)=\int_{0}^{z} \frac{c}{H(z)} \mathrm{d} z$, Where the distance measures are related to each other according to
$D_{L}=(1+z) D_{M}=(1+z)^{2} D_{A}$.
Table 1. Three polynomial parametrisations (models 1, 2, and 3) adopted in this study to place cosmology-independent constraints on $r_{\mathrm{d}}$ and $H_{0}$.

\begin{tabular}{lc}
\hline \hline Model & Formula \\
\hline 1 & $H(z)=H_{0}\left(1+b_{1} z+b_{2} z^{2}\right)$ \\
2 & $D_{L}(z)=\frac{c \ln (10)}{H_{0}}\left(\log (1+z)+c_{2}[\log (1+z)]^{2}\right.$ \\
& $\left.+c_{3}[\log (1+z)]^{3}+c_{4}[\log (1+z)]^{4}\right)$ \\
3 & $D_{M}(z)=\frac{c}{H_{0}}\left(\frac{z}{1+z}+d_{2}\left[\frac{z}{1+z}\right]^{2}+d_{3}\left[\frac{z}{1+z}\right]^{3}+d_{4}\left[\frac{z}{1+z}\right]^{4}\right)$ \\
4 & $H(z)=H_{0} \sqrt{\Omega_{M}(1+z)^{3}+\Omega_{\Lambda}+\Omega_{k}(1+z)^{2}}$ \\
\hline
\end{tabular}

Notes. The fourth case is a $\Lambda$ CDM cosmological model.

and for models 2 and $3, H(z)$ is obtained through

$H\left(z, \Omega_{k}\right)=\frac{\mathrm{c}}{\partial D_{M}(z) / \partial z} \sqrt{1+\frac{H_{0}^{2} \Omega_{k}}{\mathrm{c}^{2}} D_{\mathrm{M}}(z)^{2}}$

(Weinberg 1972).

We truncated all polynomials at the lowest expansion order required by the condition that models 1,2 , and 3 recover distances in a $\Lambda C D M$ model, if their free coefficients are fixed at values found by Taylor expanding the corresponding functions in the fiducial $\Lambda$ CDM model (see more in Appendix B). This guarantees that expansion histories derived from the employed models converge to $\Lambda \mathrm{CDM}$ once observations become consistent exclusively with the standard model. Distances in $\Lambda \mathrm{CDM}$ are recovered with a minimum accuracy of two percent at $z<1.8$, where the accuracy limit is set by the current precision of the Hubble constant measurements and the upper limit of redshift is given by the most distant lensed quasar. Including higher order terms is disfavoured by the Bayesian information criterion (BIC). In Appendix B, we also show that this convergence criterion ensures that biases in $H_{0}$ are at a sub-percent level, and biases in $q_{0}$ at a few-percent level.

Finally, in order to compare models 1-3 with the most commonly adopted cosmological model, the fourth family (model 4) adopts a $\Lambda \mathrm{CDM}$ parametrisation. In all cases, both flatness and departures from it are considered.

\subsection{Inference}

We fitted four models listed in Table 1 to observational data of type Ia supernovae, BAO, and lensed quasars. Constrained model parameters include $r_{\mathrm{d}}, H_{0}$ and all remaining free polynomial coefficients (or density parameters in the case of a $\Lambda \mathrm{CDM}$ model). The posterior distributions of the parameters were obtained using affine-invariant Monte Carlo Markov chains (MCMC; Goodman \& Weare 2010), and in particular the python module emcee (Foreman-Mackey et al. 2013). For the sake of completeness, we also derived constraints on the deceleration parameter $q_{0}$ using the MCMC samples. Appendix B outlines the relations between polynomial coefficients, which are primary parameters in our fits, and $q_{0}$.

The likelihoods of the distances measured from lensed quasars were either given as a skewed log-normal distribution $^{2}$ (for B1608) or as samples of points from the H0LiCOW model posteriors (for RXJ1131, HE0435, PG1115, J1206, and

2 Full names and coordinates of each lens are given in the H0LiCOW XIII paper (Wong et al. 2020). 
WFI2033). The probability density was obtained by constructing a Gaussian kernel density estimator (KDE). For the lens systems HE0435 and WFI2033, only a robust measurement of their time-delay distance ${ }^{3}$ was provided, which is the only robust distance currently derived from time-delay lensing in the presence of significant perturbers at lower redshift. For the remaining four lenses (B1608, RXJ1131, PG1115, J1206), information on both their time-delay distances and their angular diameter distances was available. For the remaining observables (BAO, SNe, quasars, and SHOES or CCHP), the general form of the likelihood for each data set is given by

$\mathcal{L}=p($ data $\mid$ model $) \propto e^{-\chi^{2} / 2}$,

$\chi^{2}=\mathbf{r}^{\dagger} \mathbf{C}^{-1} \mathbf{r}$,

where $\mathbf{C}$ is the covariance matrix of the data, and $\mathbf{r}$ corresponds to the difference between the predicted and the observed values. The final likelihood is a product of the separate likelihoods corresponding to each data set.

A uniform prior was used for the parameters, for ease of comparison with previous works. In particular, the value of $r_{\mathrm{d}}$ was kept between 0 and $200 \mathrm{Mpc}$ and, if applicable, $\Omega_{k}$ between -1 and 1 and $\Omega_{\mathrm{m}}$ between 0.05 and 0.5 , to ensure consistency with the priors on $\Omega_{\mathrm{m}}$ by HOLiCOW. These priors do not skew the inference, at least with the current uncertainties. The upper and lower boundaries of $r_{\mathrm{d}}$ do not influence any of the results. For the coefficients of the expansion $\left(b_{i}, c_{i}\right.$, and $d_{i}$ in Table 1$)$, we used a uniform prior without limits. In all cases, best fit values are given by the posterior mean and errors provide 68.3 percent confidence intervals. The code to generate the results in this paper is publicly available on Github ${ }^{4}$.

\section{Comparison with the CMB: data and models}

The sound horizon and the Hubble constant are independently measured from the CMB. For the standard flat $\Lambda \mathrm{CDM}$ cosmological model, the Planck observations yield $r_{\mathrm{d}}=147.2 \pm 0.3 \mathrm{Mpc}$ and $H_{0}=67.4 \pm 0.5 \mathrm{~km} \mathrm{~s}^{-1} \mathrm{Mpc}^{-1}$ (Planck Collaboration VI 2020). As we demonstrate, both parameters are strongly discrepant with their counterparts determined from late-time observations. In the following subsections, we describe how we quantified this tension, and we outline a few popular extensions of the standard cosmological model devised to reduce the discrepancy.

\subsection{Quantifying the tension}

In order to check whether or not our results for $r_{\mathrm{d}}$ and $H_{0}$ are in agreement with those obtained by Planck, the Gaussian odds indicator $\tau$ is used (Verde et al. 2013; Bernal et al. 2016):

$\tau=\frac{\int \bar{P}_{A} \bar{P}_{B} \mathrm{~d} x}{\int P_{A} P_{B} \mathrm{~d} x}$.

Here, $P_{A}$ and $P_{B}$ denote the posterior distributions of experiments $A$ and $B$, while $\bar{P}_{A}$ and $\bar{P}_{B}$ correspond to the same distributions after a shift has been performed, such that the maxima of $P_{A}$ and $P_{B}$ coincide. A high value for $\tau$ means that it is unlikely that both experiments measure the same quantity. In an idealised

\footnotetext{
$3 D_{\Delta t}=\left(1+z_{\mathrm{l}}\right) D_{\mathrm{A}, \mathrm{l}} D_{\mathrm{A}, \mathrm{s}} / D_{\mathrm{A}, \mathrm{ls}}$, where $D_{\mathrm{A}, \mathrm{l}}, D_{\mathrm{A}, \mathrm{s}}$ and $D_{\mathrm{A}, \mathrm{ls}}$ are the angular distance to the lens galaxy, lensed quasar, and between the lens and the quasar; $z_{1}$ is redshift of the lens galaxy.

4 https://github.com/Nikki1510/cosmic_dissonance
}

situation, when experiment $A$ yields a measurement with infinite precision ( $P_{A}$ is given a $\delta$ function), the odds indicator equals the ratio of probability $P_{B}$ evaluated at best fit values returned by both experiments. Equation (5) generalises this interpretation to cases where both measurements have non-zero uncertainties.

A more intuitive scale representing the discrepancy between two measurements is a number-of-sigma tension, and it can be directly derived from the odds ratios (see e.g. Bernal et al. 2016). First, the odds indicator was used to calculate the probability enclosed by a contour $r$, such that $1 / \tau=e^{-\frac{1}{2} r^{2}}$. The probability was then converted to a number of sigma tension, using a onedimensional cumulant (the error function).

\subsection{Extensions of the $\Lambda C D M$ model}

Any tension between late-time measurements and CMB-based model-dependent inference may be caused by unknown systematics, or it can mean that our knowledge of the physics underlying the expansion history is incomplete. The standard flat $\Lambda C D M$ model can be extended either by changing physics in the early Universe (pre-recombination; this is referred to as early-time modification) or at later epochs (post-recombination; this is referred to as late-time modification). In the first case, one can decrease the sound horizon inferred from the CMB observations by adding an energy-momentum tensor beyond the Standard Model, which effectively increases $H(z)$ in the early Universe. In order to keep the observed angular scales imprinted in the CMB unchanged, this alteration automatically implies an increase in the value of $H_{0}$. Therefore, the overall effect of earlytime modifications is a shift of both $r_{\mathrm{d}}$ and $H_{0}$ towards the measurements from late-time observations. In the second approach, one may obtain higher values of $H_{0}$ by decreasing the expansion rate at intermediate redshifts. This can be done by modifying the dark energy density such that it increases over time. Although many late-time extensions of the Standard Model can quite easily increase $H_{0}$ inferred from the CMB, $r_{\mathrm{d}}$ cannot be modified as appreciably as $H_{0}$ - as it is primarily driven by physics in the early Universe.

In order to explore different resolutions of the tension in $H_{0}$ and $r_{\mathrm{d}}$ on the grounds of new physics, we considered several extensions of the standard $\Lambda \mathrm{CDM}$ model. Although the selected models do not exhaust all possible proposals from the literature, they are sufficiently representative in terms of covering most possible model-dependent alterations of $H_{0}$ and $r_{\mathrm{d}}$ inferred from the CMB. In what follows, the inference for early dark energy and PEDE (described below) were obtained using a Planck compressed likelihood, as detailed in Appendix A. For the remaining models, we used publicly available MCMC chains (based on Planck temperature and polarisation data) from the Planck Legacy Archive ${ }^{5}$ (Planck Collaboration VI 2020).

\subsubsection{Early-time (pre-recombination) extensions}

Effective number of relativistic species $\left(N_{\mathrm{eff}}\right)$. In this extension of $\Lambda \mathrm{CDM}$, there are additional relativistic particles that contribute to the radiation density of the early Universe, resulting in $N_{\text {eff }}>3$. An increased radiation density leads to a later matter-radiation equality and to an increased expansion rate in the early Universe, leaving an observational imprint on the CMB (Eisenstein \& White 2004; Hannestad 2003; Mörtsell \& Dhawan 2018). This in turn reduces the value of the sound horizon $r_{\mathrm{d}}$ at recombination and increases $H_{0}$ derived from the $\mathrm{CMB}$, thereby relieving some of the tension

\footnotetext{
5 https://pla.esac.esa.int
} 
Table 2. Posterior mean and standard deviation for the sound horizon $r_{\mathrm{d}}, H_{0} r_{\mathrm{d}}$ and $q_{0}$ inferred from late-time observations including H0LiCOW lensing observations, Pantheon SN sample, and BAO measurements (BOSS).

\begin{tabular}{|c|c|c|c|c|}
\hline \multirow[b]{2}{*}{ Parameter } & \multicolumn{4}{|c|}{ Flat $\left(\Omega_{\mathrm{k}}=0\right)$} \\
\hline & Model 1 & Model 2 & Model 3 & Model $4(f \Lambda \mathrm{CDM})$ \\
\hline$r_{\mathrm{d}}(\mathrm{Mpc})$ & $132.7 \pm 4.2$ & $132.9 \pm 4.4$ & $134.2 \pm 4.4$ & $136.9 \pm 3.7$ \\
\hline$H_{0} r_{\mathrm{d}}\left(\mathrm{km} \mathrm{s}^{-1}\right)$ & $10107 \pm 147$ & $10065 \pm 150$ & $10052 \pm 152$ & $10038 \pm 136$ \\
\hline$q_{0}$ & $-0.7 \pm 0.07$ & $-0.5 \pm 0.2$ & $-0.4 \pm 0.3$ & $-0.55 \pm 0.03$ \\
\hline $\ln \mathcal{L}_{\text {m.a.p. }}$ & -86.3 & -86.1 & -86.7 & -87.7 \\
\hline BIC score & 193 & 196 & 198 & 192 \\
\hline $\ln \tau($ Planck $\Lambda \mathrm{CDM})$ & $6.6(3.2 \sigma)$ & $5.7(2.9 \sigma)$ & $5.0(2.7 \sigma)$ & $5.7(2.9 \sigma)$ \\
\hline $\ln \tau\left(\right.$ Planck $\left.\Lambda \mathrm{CDM}+N_{\mathrm{eff}}\right)$ & $6.3(3.1 \sigma)$ & $5.6(2.9 \sigma)$ & $4.9(2.7 \sigma)$ & $5.0(2.7 \sigma)$ \\
\hline \multirow[t]{2}{*}{$\ln \tau$ (Planck early DE) } & $5.1(2.8 \sigma)$ & $4.4(2.5 \sigma)$ & $3.7(2.3 \sigma)$ & $3.7(2.2 \sigma)$ \\
\hline & \multicolumn{4}{|c|}{ Free $\Omega_{\mathrm{k}}$} \\
\hline Parameter & Model 1 & Model 2 & Model 3 & Model $4(\Lambda \mathrm{CDM})$ \\
\hline$r_{\mathrm{d}}(\mathrm{Mpc})$ & $129.2 \pm 5.7$ & $130.6 \pm 5.9$ & $131.2 \pm 6.1$ & $137.2 \pm 4.8$ \\
\hline$H_{0} r_{\mathrm{d}}\left(\mathrm{km} \mathrm{s}^{-1}\right)$ & $10045 \pm 155$ & $10033 \pm 157$ & $10017 \pm 160$ & $10041 \pm 156$ \\
\hline$\Omega_{k}$ & $0.18 \pm 0.2$ & $0.13 \pm 0.2$ & $0.15 \pm 0.2$ & $-0.01 \pm 0.2$ \\
\hline & $-0.6 \pm 0.1$ & $-0.4 \pm 0.2$ & $-0.4 \pm 0.3$ & $-0.56 \pm 0.07$ \\
\hline $\ln \mathcal{L}_{\text {m.a.p. }}$ & -86.1 & -85.9 & -86.4 & -87.7 \\
\hline BIC score & 196 & 200 & 201 & 196 \\
\hline $\ln \tau($ Planck $\Lambda \mathrm{CDM})$ & $5.6(2.9 \sigma)$ & $4.6(2.6 \sigma)$ & $4.0(2.4 \sigma)$ & $4.2(2.4 \sigma)$ \\
\hline $\ln \tau\left(\right.$ Planck $\left.\Lambda \mathrm{CDM}+N_{\mathrm{eff}}\right)$ & $5.7(2.9 \sigma)$ & $4.7(2.6 \sigma)$ & $4.2(2.4 \sigma)$ & $3.9(2.3 \sigma)$ \\
\hline $\ln \tau$ (Planck early DE) & $4.5(2.6 \sigma)$ & $3.6(2.2 \sigma)$ & $3.1(2.0 \sigma)$ & $2.6(1.8 \sigma)$ \\
\hline
\end{tabular}

Notes. The fit quality is summarised in terms of log-likelihood at the maximum posterior probability, $\ln \mathcal{L}_{\text {m.a.p. }}$, and the Bayesian information criterion BIC $=\ln (N) k-2 \ln \left(\mathcal{L}_{\text {m.a.p. }}\right)$, where $N$ is the number of data points and $k$ is the number of free parameters. The odds indicator $\tau$ quantifies the tension between $r_{\mathrm{d}}$ and $H_{0}$ measured from late-time observations and the Planck data (for the standard flat $\Lambda$ CDM model and its two extensions with a free effective number of neutrinos or early dark energy).

between late-time and $\mathrm{CMB}$ measurements (Carneiro et al. 2019; Gelmini et al. 2019).

Early dark energy. The expansion rate in the early Universe could also be increased by the presence of a more general form of dark energy. This additional dark energy should have a noticeable contribution to the energy budget at high redshifts, but should dilute away faster than radiation to leave the evolution of the Universe after recombination unchanged (Doran et al. 2007; Linder \& Robbers 2008). As a promising example of this class of models, we considered early dark energy, which behaves nominally as a scalar field $\phi$ with a potential $V(\phi) \propto[1-\cos (\phi / f)]^{3}$ (Poulin et al. 2019). In the effective fluid description, the energy density $\rho_{\mathrm{EDE}}$ evolves as

$\rho_{\mathrm{EDE}}(a)=\frac{2 \rho_{\mathrm{EDE}}\left(a_{\mathrm{c}}\right)}{1+\left(a / a_{\mathrm{c}}\right)^{9 / 2}}$,

with the scale factor $a$ (Poulin et al. 2018). The early dark energy equation of state approaches asymptotically -1 for $a \ll a_{c}$ and $1 / 2$ for $a \gg a_{c}$. When fitting the model to the CMB data, we adopted the following flat priors in $\log _{10}\left(a_{\mathrm{c}}\right)$ and $f_{\mathrm{EDE}}=$ $\Omega_{\phi}\left(a_{\mathrm{c}}\right) / \Omega_{\mathrm{tot}}\left(a_{\mathrm{c}}\right):-4.0<\log _{10}\left(a_{\mathrm{c}}\right)<-3.2$ and $0.1>f_{\mathrm{EDE}}>0$.

\subsubsection{Late-time (post-recombination) extensions}

Time-dependent dark energy (wCDM). The $w C D M$ cosmology introduces the equation of state parameter $w$ as a free parameter (as opposed to the fixed $\Lambda$ CDM value of $w=-1$ ), so that the dark energy density $\rho_{\mathrm{DE}}$ can change as a function of redshift as

$\rho_{\mathrm{DE}}(z)=\rho_{\mathrm{DE}, 0}(1+z)^{3(1+w)}$.

Phenomenologically emergent dark energy (PEDE). In the PEDE model, dark energy has no effective role in the early
Universe but emerges at later times (Li \& Shafieloo 2019). The redshift evolution of the dark energy density is described by

$\rho_{\mathrm{DE}}(z)=\rho_{\mathrm{DE}, 0} \times\left[1-\tanh \left(\log _{10}(1+z)\right)\right]$,

giving it the same number of degrees of freedom as $\Lambda \mathrm{CDM}$. We emphasise that this parametrisation is mostly ad hoc.

\section{Results and discussion}

The values of the sound horizon and other parameters inferred from the six lenses, Pantheon SN sample, and BAO measurements (BOSS) using three models that employ polynomial parametrisation or a $\Lambda \mathrm{CDM}$ model are listed in Table 2. The tension with Planck flat $\Lambda \mathrm{CDM}$ and late-time extension models is displayed in the last rows and ranges from $2 \sigma$ to $3 \sigma$. When combining the distance calibration from the lensed quasars with that from SHOES (the distance ladder with Cepheids), the constraints on $r_{\mathrm{d}}$ are tighter and the tension with Planck increases to $5 \sigma$, as can be seen in Table 3. The corresponding Bayesian information criterion (BIC) values are the lowest for model 4 $(\Lambda \mathrm{CDM})$. However, the differences in BIC scores do not exceed six (substantial level on the Jeffreys scale), with a minimum difference of one for model 1 (barely worth mentioning level on the Jeffreys scale). Figure 1 compares constraints on $H_{0}, r_{\mathrm{d}}$ and $\Omega_{\mathrm{k}}$ from late-time observations including the prior from SHOES to the best fit parameters derived from Planck assuming a flat $\Lambda \mathrm{CDM}$ model. For all models, the Planck parameters lie on the $5 \sigma$ contour in the $H_{0}-r_{\mathrm{d}}$ plane, demonstrating that the tension is independent of the chosen expansion family.

In Table 4, some other combinations of data sets are explored. This includes a calibration of lenses + CCHP instead of SHOES, inclusion of several volume-averaged and Ly- $\alpha$ BAO 
Table 3. Same as Table 2, but for fits based on the HOLiCOW lensing, Pantheon SN sample, BAO measurements (BOSS) and $H_{0}$ from SHOES.

\begin{tabular}{|c|c|c|c|c|}
\hline \multirow[b]{2}{*}{ Parameter } & \multicolumn{4}{|c|}{ Flat $\left(\Omega_{\mathrm{k}}=0\right)$} \\
\hline & Model 1 & Model 2 & Model 3 & Model $4(f \Lambda C D M)$ \\
\hline$r_{\mathrm{d}}(\mathrm{Mpc})$ & $135.1 \pm 2.8$ & $135.0 \pm 2.9$ & $135.1 \pm 2.9$ & $136.1 \pm 2.7$ \\
\hline$H_{0} r_{\mathrm{d}}\left(\mathrm{km} \mathrm{s}^{-1}\right)$ & $10079 \pm 143$ & $10055 \pm 148$ & $10038 \pm 153$ & $10037 \pm 136$ \\
\hline$q_{0}$ & $-0.6 \pm 0.07$ & $-0.4 \pm 0.2$ & $-0.4 \pm 0.3$ & $-0.55 \pm 0.03$ \\
\hline $\ln \mathcal{L}_{\text {m.a.p. }}$ & -86.6 & -86.4 & -86.8 & -87.7 \\
\hline BIC score & 193 & 197 & 198 & 192 \\
\hline $\ln \tau($ Planck $\Lambda$ CDM $)$ & $15.1(5.1 \sigma)$ & $15.0(5.1 \sigma)$ & $13.9(4.9 \sigma)$ & $15.1(5.1 \sigma)$ \\
\hline $\ln \tau\left(\right.$ Planck $\left.\Lambda \mathrm{CDM}+N_{\mathrm{eff}}\right)$ & $9.9(4.1 \sigma)$ & $9.7(4.0 \sigma)$ & $9.2(3.9 \sigma)$ & $9.1(3.9 \sigma)$ \\
\hline \multirow[t]{2}{*}{$\ln \tau$ (Planck early DE) } & $9.4(3.9 \sigma)$ & $9.2(3.9 \sigma)$ & $8.6(3.7 \sigma)$ & $8.7(3.8 \sigma)$ \\
\hline & \multicolumn{4}{|c|}{ Free $\Omega_{\mathrm{k}}$} \\
\hline Parameter & Model 1 & Model 2 & Model 3 & Model $4(\Lambda \mathrm{CDM})$ \\
\hline$r_{\mathrm{d}}(\mathrm{Mpc})$ & $134.8 \pm 3.2$ & $134.7 \pm 3.3$ & $134.6 \pm 3.3$ & $136.1 \pm 3.2$ \\
\hline$H_{0} r_{\mathrm{d}}\left(\mathrm{km} \mathrm{s}^{-1}\right)$ & $10067 \pm 156$ & $10042 \pm 161$ & $10021 \pm 161$ & $10035 \pm 152$ \\
\hline$\Omega_{k}$ & $0.04 \pm 0.2$ & $0.03 \pm 0.2$ & $0.06 \pm 0.2$ & $0.003 \pm 0.2$ \\
\hline$q_{0}$ & $-0.6 \pm 0.09$ & $-0.4 \pm 0.2$ & $-0.4 \pm 0.3$ & $-0.55 \pm 0.07$ \\
\hline $\ln \mathcal{L}_{\text {m.a.p. }}$ & -86.7 & -86.5 & -86.8 & -87.7 \\
\hline BIC score & 198 & 201 & 202 & 196 \\
\hline $\ln \tau($ Planck $\Lambda \mathrm{CDM})$ & $13.3(4.8 \sigma)$ & $13.2(4.8 \sigma)$ & $12.7(4.7 \sigma)$ & $12.8(4.7 \sigma)$ \\
\hline $\ln \tau\left(\right.$ Planck $\left.\Lambda \mathrm{CDM}+N_{\mathrm{eff}}\right)$ & $9.2(3.9 \sigma)$ & $9.0(3.9 \sigma)$ & $8.9(3.8 \sigma)$ & $8.2(3.6 \sigma)$ \\
\hline $\ln \tau$ (Planck early DE) & $8.3(3.7 \sigma)$ & $8.2(3.7 \sigma)$ & $8.0(3.6 \sigma)$ & $7.3(3.4 \sigma)$ \\
\hline
\end{tabular}

and the addition of high redshift quasars as secondary standard candles. Considering all results based on the main data sets (HOLiCOW, SN, BAO/BOSS) with the cosmic distance ladder (SH0ES or CCHP), we find $r_{\mathrm{d}}=\left(137 \pm 3^{\text {stat. }} \pm 2^{\text {syst. }}\right)$ Mpc, where the systematic error accounts for differences between SHOES and CCHP distance calibration. In addition, we ran an inference free of any $\mathrm{SN}$ data, thus only using lensed quasars and BAO measurements from BOSS, $D_{V}$ and Ly- $\alpha$ with a flat $\Lambda$ CDM model $^{6}$ This results in the following values for the cosmological parameters: $r_{\mathrm{d}}=138.6 \pm 3.8 \mathrm{Mpc}, H_{0} r_{\mathrm{d}}=10166 \pm 142 \mathrm{~km} \mathrm{~s}^{-1}$, $\Omega_{\mathrm{m}}=0.29 \pm 0.02$.

\subsection{Early-time extensions}

A possible solution for the tension is an extension to the early Universe physics, such as an additional component of relativistic species. Planck 2018 chains with free $N_{\text {eff }}$ (based on full temperature and polarisation data) were used to investigate this scenario. In Fig. 2, Planck + free $N_{\text {eff }}$ is compared to results from model 3 using SN + BAO with only the HOLiCOW lenses as calibrator (upper panel) and using a combination of HOLiCOW lenses and either SHOES or CCHP as calibrators (lower panel). A higher value of $N_{\text {eff }}$ is shown to move the Planck value to a lower $r_{\mathrm{d}}$ and a higher $H_{0}$, therefore alleviating the tension to some extent. In this case, the combined analysis of Planck and low-redshift data yields $N_{\text {eff }}=3.24 \pm 0.16$. This effect is only convincing when the late-time measurements are calibrated with HOLiCOW and CCHP, since the alternative Cepheid calibration is still in tension with the Planck $+N_{\text {eff }}$ extension (see Table 3).

\subsection{Tension between the $C M B$ and late-time observations}

Figure 3 demonstrates the potential of the selected extensions of the standard $\Lambda \mathrm{CDM}$ model outlined in Sect. 3.2 to resolve

\footnotetext{
${ }^{6}$ For the flat $\Lambda \mathrm{CDM}$ model, we adopted a prior of $\Omega_{\mathrm{M}}=\mathcal{U}[0.05,0.5]$.
}

the tension between $r_{\mathrm{d}}$ and $H_{0}$ measured from the CMB and late-time observations. The shaded grey contours show constraints from late-time observations using model 3 with $\Omega_{\mathrm{k}}=0$. Thanks to a polynomial parametrisation, these measurements are marginalised over a wide class of the expansion history and in this sense they are independent of cosmological model. We show results for distance calibrations based on the H0LiCOW lenses combined with SHOES or CCHP. The contours in colour show constraints from Planck for the flat $\Lambda \mathrm{CDM}$ model (black contours) and its four extensions.

As clearly seen from Fig. 3, none of the $\Lambda \mathrm{CDM}$ extensions manage to convincingly unify the Planck measurements with the late-time ones if the SHOES calibration is used to anchor the distance ladder. In particular, late-time extensions involving different generalisations of the cosmological constant can increase the $H_{0}$ value inferred from the CMB, but they leave $r_{\mathrm{d}}$ unchanged. Although early-time extensions can potentially match both $H_{0}$ and $r_{\mathrm{d}}$ from low-redshift probes and the $\mathrm{CMB}$, that this may happen by expanding the posterior probability contours rather than shifting the best fit values (see also Bernal et al. 2016; Karwal \& Kamionkowski 2016), as demonstrated in Fig. 3. In this respect, both early dark energy models and extensions with extra relativistic species are quite similar. The apparent difference between their probability contours reflect differences in the priors. While a free effective number of relativistic species can either decrease or increase the sound horizon, early dark energy (with positive energy density) can only increase the energy budget, and thus decrease the sound horizon.

Figure 4 summarises the tension in the $H_{0}-r_{\mathrm{d}}$ plane between late-time measurements and Planck with different extensions of $\Lambda \mathrm{CDM}$. To ensure a fair comparison, the same $\Lambda \mathrm{CDM}$ extensions are used in the late-time and CMB-based inference. Therefore, the Planck PEDE-CDM results have been compared to late-time results obtained with PEDE-CDM, and the Planck wCDM results to late-time results using $w C D M$. For the earlytime extensions, this is not of great importance, since their effects do not influence the low-redshift measurements. 

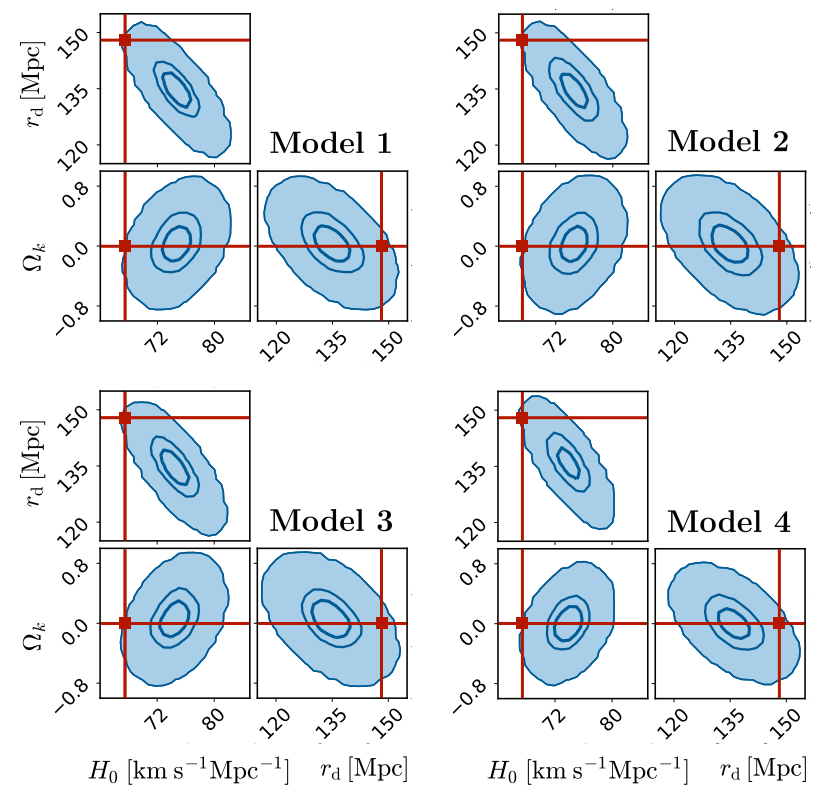

Fig. 1. Constraints on sound horizon $r_{\mathrm{d}}$, Hubble constant $H_{0}$ and $\Omega_{\mathrm{k}}$ from late-time observations including BAO (BOSS), type Ia supernovae (Pantheon), gravitational lensing (HOLiCOW) and cosmic distance ladder calibrated with Cepheids (SHOES). The panels show results for three cosmology-independent models listed in Table 1 and a $\Lambda$ CDM cosmological model. The red lines indicate the best fit values obtained from Planck for a flat $\Lambda \mathrm{CDM}$ cosmological model. The contours indicate 1-, 2- and $5 \sigma$ confidence regions of the posterior probability (the latter obtained by Gaussian extrapolation). All panels demonstrate a $5 \sigma$ tension between $r_{\mathrm{d}}$ and $H_{0}$ measured from the CMB and the late-time observations.

By adopting different models of polynomial parametrisations (models 1,2, and 3), we minimised the dependence on a cosmological model. Although our inference with these models does not depend on $\Lambda \mathrm{CDM}$, it does have a weak dependency on general relativity (GR). The lensed quasars that are used to calibrate the distance ladder need GR in order to calculate the angular diameter distance, through the Ansatz that the lensing potential (used in the time-delay inference) is exactly twice the gravitational potential (used to obtain $D_{A} \propto \mathrm{c}^{3} \Delta t / \sigma^{2}$ from stellar kinematics). However, the role of this GR dependence is subdominant with current $D_{A}$ uncertainties $(10 \%-20 \%)$. On the other hand, GR also enters the early-Universe expansion through the "abundances" of different components $\left(\Omega_{\mathrm{m}} \Omega_{\mathrm{de}}, N_{\mathrm{eff}}\right)$.

\subsection{One lens at a time}

Since $H_{0}$ and $r_{\mathrm{d}}$ are constants, they must be independent of the chosen indicators. If they are inferred from each indicator separately, any trend will signal residual systematics, either in the indicators themselves or in the parametrisation that is chosen to extrapolate $H(z)$ down to $H_{0}$.

The HOLiCOW collaboration has shown that if $H_{0}$ is obtained from lenses in a flat- $\Lambda C D M$ model, there is a weak trend in its inferred value versus redshift. Lenses of lower (higher) redshift differ more (less) from the Planck measurements (Wong et al. 2020). Even though this trend is currently not significant (given current uncertainties), it may be indicative of intrinsic systematics in the lensing inference, or in the way that time-delay distances are converted into $H_{0}$ values through a flat- $\Lambda C D M$ parametrisation.

Here, we repeat this test using more general models of the expansion history, specifically flat model 3 and flat PEDE-CDM model. Figure 5 shows the sound horizon $r_{\mathrm{d}}$ measured from combining BAO and SNe data with lensing constraints from each lens separately. The results demonstrate that the distance calibration from HOLiCOW lenses shows a similar trend with lens redshift as the one shown by Wong et al. (2020) for a flat $\Lambda \mathrm{CDM}$ cosmology. Based on the sample-wide analysis by Millon et al. (2020), this weak trend cannot be explained simply on the basis of known systematics in the lens models or kinematics of each lens. We should emphasise, however, that this trend is not statistically significant $(1.6 \sigma)$ yet.

Although the current weak trend of $r_{\mathrm{d}}$ with redshift of gravitatonal lens is consistent with being a statistical fluke, it is instructive to investigate if there any expansion models that can re-absorb this (weak) trend. For example, a recent $(z \approx 0.4)$ change in dark energy may produce this behaviour, if the data are interpreted with expansion histories that are "too" smooth. For this reason, we examined the same lens-by-lens determination within the PEDE model family. The results are shown as dotted error-bars in Fig. 5. Even the PEDE model with accelerated late-time expansion cannot eliminate the (weak) trend in $r_{\mathrm{d}}$. The constraints set by the relative distance moduli of SN enforce PEDE to closely resemble the $\Lambda$ CDM case, but with a higher matter content $\left(\Omega_{\mathrm{m}} \approx 0.345\right)$ and smaller sound horizon $\left(r_{\mathrm{d}} \approx 138 \mathrm{Mpc}\right)$. Therefore, PEDE does not resolve the current tension.

\section{Conclusions and outlook}

We combined the newest available low-redshift probes to obtain an estimate of the sound horizon at the drag epoch, $r_{\mathrm{d}}$. In order to minimise the dependence on a cosmological model, we used a set of polynomial parametrisations that are almost entirely independent of the underlying cosmology, as well as the standard $\Lambda$ CDM model. In the $H_{0}-r_{\mathrm{d}}$ plane, we found a tension of $5 \sigma$ between Planck results using flat $\Lambda \mathrm{CDM}$ and late-time observations calibrated with HOLiCOW lenses and SHOES. This tension is reduced to $2.4 \sigma$ if CCHP results are used as a distanceladder anchor instead of SHOES. We investigated whether earlyor late-time extensions to the standard $\Lambda \mathrm{CDM}$ model can resolve the tension, and we examined models with free $N_{\text {eff }}$, early dark energy, $w \mathrm{CDM}$, and PEDE-CDM. None of these model extensions provide a satisfying solution to the Hubble tension problem (see also Aylor et al. 2019; Knox \& Millea 2020), except for free $N_{\text {eff }}$ or early dark energy in combination with low redshift data calibrated by $\mathrm{CCHP}+\mathrm{HOLiCOW}$.

These findings may indicate that: (1) extensions of earlytime physics are necessary; and/or (2) that systematics from different late-time probes are becoming comparable to the statistical uncertainties. Arguments based on local under-densities or peculiar velocities cannot resolve the tension: the $\approx 3 \sigma$ tension persists if the inverse-distance ladder is restricted to $z \geq$ 0.03 , where the role of peculiar velocities is $\lesssim 0.1 \%$ (see also Wojtak \& Agnello 2019). Multiple secondary sources of errors in redshift measurements were studied by Davis et al. (2019), but none of them seem to have any noticeable effect. Another explanation may be that the standardisation of SNe Ia is not properly understood yet (as a caveat, see Rigault et al. 2015, for example, or Khetan et al. 2020), or that there is some (hitherto undiscovered) source of systematics in one of the other used data sets. If all astrophysical systematics are exhausted, one can also consider proposals involving non-standard physics in the local Universe such as screened fifth forces, which may bias $H_{0}$ measurements high via modulation of gravity-dependent pulsation periods of Cepheids (for more details see Desmond et al. 2019). 
Table 4. Same as Table 2, but for various combinations of late-time observations including two local determinations of $H_{0}$ (SH0ES or CCHP), measurements of isotropic BAO $\left(D_{\mathrm{V}}\right)$ and anisotropic BAO from the Lyman- $\alpha$ forest of quasars (Ly- $\left.\alpha\right)$, and estimates of distance moduli from high-redshift quasars.

\begin{tabular}{|c|c|c|c|c|}
\hline \multirow[b]{2}{*}{ Parameter } & \multicolumn{4}{|c|}{ Flat $\left(\Omega_{\mathrm{k}}=0\right)$} \\
\hline & $\begin{array}{l}\mathrm{CCHP}+\mathrm{HOLiCOW} \\
+\mathrm{SN}+\mathrm{BAO}(\mathrm{BOSS})\end{array}$ & $\begin{array}{l}\text { SH0ES + H0LiCOW } \\
+\mathrm{SN}+\mathrm{BAO} \text { (BOSS } \\
\left.+D_{\mathrm{V}}+\mathrm{Ly}-\alpha\right)\end{array}$ & $\begin{array}{l}\mathrm{H} 0 \mathrm{LiCOW}+\mathrm{SN}+ \\
\mathrm{BAO}\left(\mathrm{BOSS}+D_{\mathrm{V}}+\right. \\
\mathrm{Ly}-\alpha)\end{array}$ & $\begin{array}{l}\text { SHOES + HOLiCOW } \\
+ \text { SN + BAO (BOSS) } \\
+ \text { high- } z \text { quasars }\end{array}$ \\
\hline$r_{\mathrm{d}}(\mathrm{Mpc})$ & $139.5 \pm 3.6$ & $138.1 \pm 2.7$ & $138.6 \pm 3.8$ & $134.0 \pm 2.8$ \\
\hline$H_{0} r_{\mathrm{d}}\left(\mathrm{km} \mathrm{s}^{-1}\right)$ & $10019 \pm 152$ & $10197.1 \pm 135$ & $10191 \pm 138$ & $10011 \pm 149$ \\
\hline$q_{0}$ & $-0.4 \pm 0.4$ & $-0.9 \pm 0.3$ & $-0.8 \pm 0.3$ & $-0.2 \pm 0.3$ \\
\hline $\ln \tau($ Planck $\Lambda \mathrm{CDM})$ & $3.8(2.3 \sigma)$ & $12.8(4.7 \sigma)$ & $4.4(2.5 \sigma)$ & $17.1(5.5 \sigma)$ \\
\hline $\ln \tau\left(\right.$ Planck $\left.\Lambda \mathrm{CDM}+N_{\mathrm{eff}}\right)$ & $3.4(2.1 \sigma)$ & $8.0(3.6 \sigma)$ & $4.2(2.4 \sigma)$ & $11.0(4.3 \sigma)$ \\
\hline $\ln \tau$ (Planck early DE) & $2.1(1.5 \sigma)$ & $7.2(3.4 \sigma)$ & $2.8(1.9 \sigma)$ & $10.8(4.3 \sigma)$ \\
\hline
\end{tabular}

Notes. The parameters are determined using model 3 with $\Omega_{\mathrm{k}}=0$.

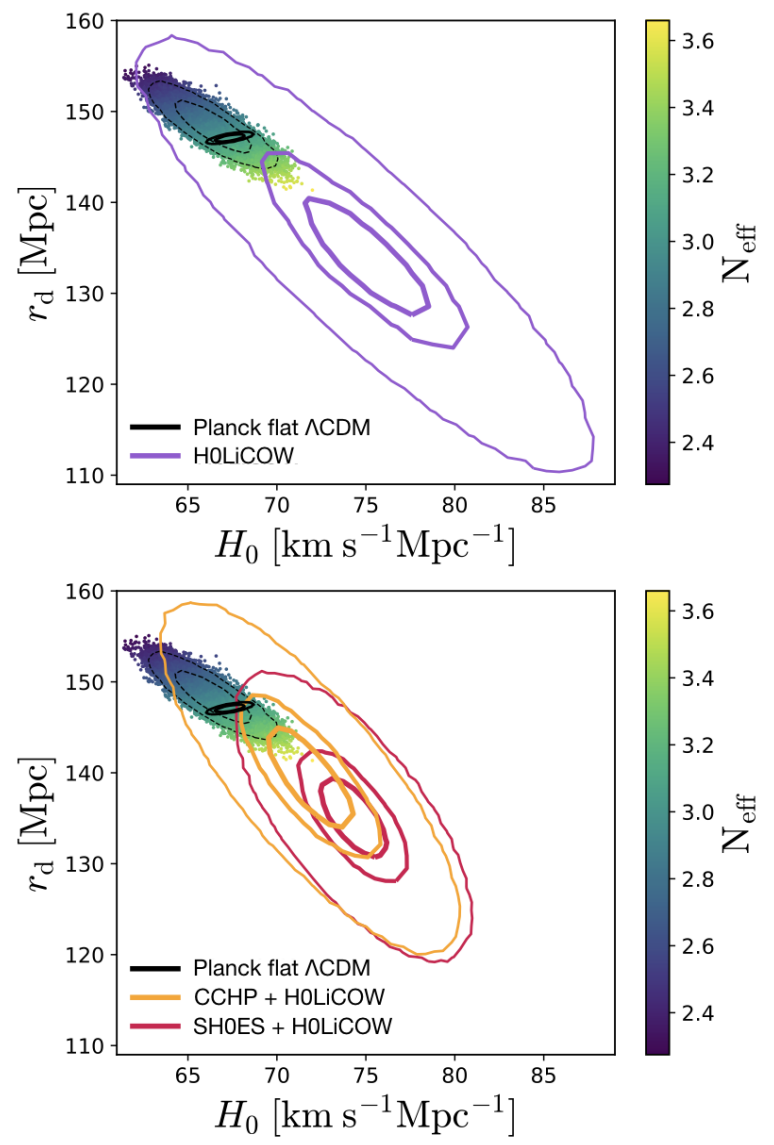

Fig. 2. Comparison between sound horizon $r_{\mathrm{d}}$ and Hubble constant $H_{0}$ measured from Planck observations of the CMB (assuming a flat $\Lambda \mathrm{CDM}$ ) and late-time observations (using flat model 3) obtained by calibrating $\mathrm{SN}$ and $\mathrm{BAO}$ measurements with three different absolute distance calibrations from: gravitational lensing (HOLiCOW), the cosmic distance ladder with Cepheids (SHOES) or the TRGB (CCHP). For the late-time data, the contours show 1-, 2- and $5 \sigma$ confidence regions of the posterior probability (the latter obtained by Gaussian extrapolation). The Planck constraints ( 1 - and $2 \sigma$ confidence regions) are obtained for the standard effective number of neutrinos (black solid line) and a model with a free effective number of neutrinos (black dashed lines, colour points).

For these reasons, we also provide a measurement that relies only on lenses and BAO, without any additional constraint from SNe, in Sect. 4.

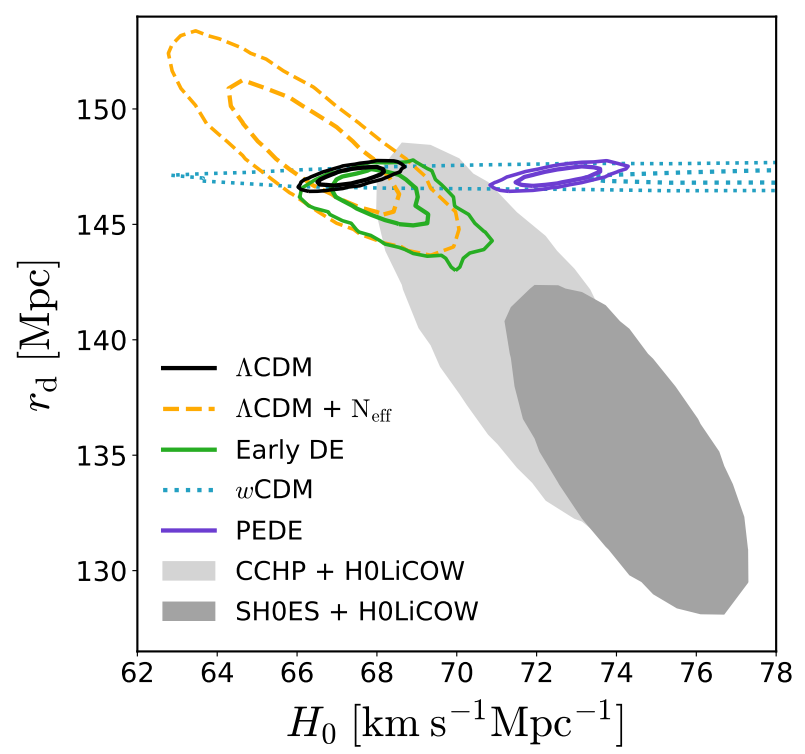

Fig. 3. Effects of four different extensions of the flat $\Lambda$ CDM model on the sound horizon and the Hubble constant measured from the Planck data. The models considered here are $\Lambda \mathrm{CDM}+$ free $N_{\text {eff }}$, early dark energy, wCDM, and PEDE. The CMB-based constraints are compared to the measurements from late-time observations $(\mathrm{SN}+\mathrm{BAO}+$ HOLiCOW + SHOES/CCHP) shown with the grey shaded contours. The late-time measurements are obtained with model 3 (see Table 1) and show the $2 \sigma$ credibility regions.

The weak trend in Fig. 5 may indicate residual systematics in the lens models, or the need for different low- $z$ expansion models, or it may vanish entirely with larger lens samples. In order to check the robustness of the trend, cosmographygrade models of more lenses are needed, over the whole $0.3 \lesssim$ $z \lesssim 0.7$ current redshift interval and beyond. Finally, the role of systematics in the lens-mass models can be assessed once high$\mathrm{S} / \mathrm{N}$ spatially resolved kinematics are available (Shajib et al. 2018; Y1ldirım et al. 2020), which would enable more flexible dynamical models than the ones used so far on aperture-averaged velocity dispersions.

As a final remark, we emphasise that resolving the $H_{0}$ tension alone is not sufficient, since different models that can shift this value are still at tension with the inferred $r_{\mathrm{d}}$ from BAO and low-redshift indicators. Also, a direct combination of the inference from late-time and CMB-based measurements that may be at $>3 \sigma$ tension, hence hardly compatible with one another, should be justified. Therefore, any new proposal to resolve the 


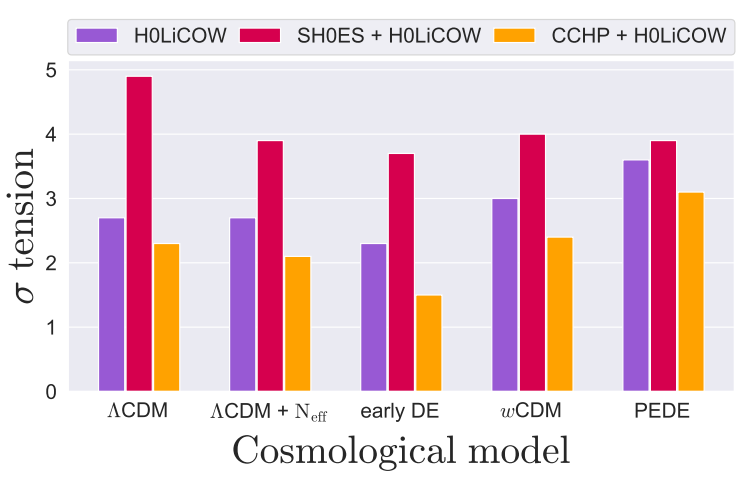

Fig. 4. Tension between sound horizon and Hubble constant measured from late-time observations and $\mathrm{CMB}$ for the following cosmological models: $\Lambda \mathrm{CDM}, \Lambda \mathrm{CDM}+N_{\text {eff }}$, early DE, wCDM, PEDE-CDM (flatness assumed in all cases). Late-time observations include BAO, type Ia supernovae, and three different absolute distance calibrations from gravitational lensing (HOLiCOW), the cosmic distance ladder with Cepheids (SHOES) or the TRGB (CCHP).

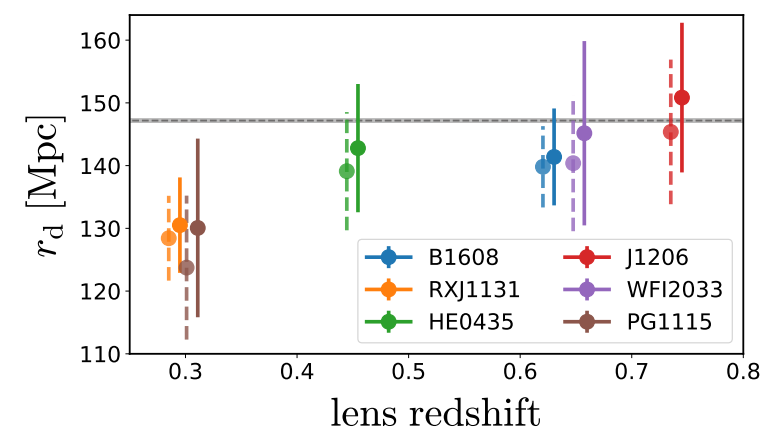

Fig. 5. Sound horizon $r_{\mathrm{d}}$ measured from combining BAO and $\mathrm{SNe}$ data with HOLiCOW lensing observations of each lens separately. Here, the distance calibration is set solely by the lensing observations of each individual lens. The measured sound horizon is shown as a function of lens redshift for fits with a flat model 3 (solid error bars) and a flat PEDECDM model (dashed error bars). For both models, the measurements show a slight trend of $r_{\mathrm{d}}$ increasing with lens redshift. The inference from models 1 and 2 is fully consistent with the model 3 results. The grey dashed line with shaded region shows Planck's value of $r_{\mathrm{d}}$ and its (sub-percent) uncertainty obtained for the standard flat $\Lambda$ CDM model.

discrepancy between CMB-based and late-time measurements should consider both $H_{0}$ and $r_{\mathrm{d}}$, and examine the separate inference upon late-time and CMB-based data.

Acknowledgements. We thank Chiara Spiniello for useful comments on an earlier version of this manuscript, and Inh Jee and Eiichiro Komatsu for discussions on the lensing distance likelihoods. These time delay cosmography observations are associated with programs HST-GO-9375, HST-GO-9744, HST-GO10158, HST-GO-12889, and HST-14254. Support for programs HST-GO-10158 HST-GO-12889 HST-14254 was provided to members of our team by NASA through grants from the Space Telescope Science Institute, which is operated by the Association of Universities for Research in Astronomy, Inc., under NASA contract NAS 5-26555. AA and RJW were supported by a grant from VILLUM FONDEN (project number 16599). This project is partially funded by the Danish council for independent research under the project "Fundamentals of Dark Matter Structures", DFF-6108-00470. This project has received funding from the European Research Council (ERC) under the EU's Horizon 2020 research and innovation programme (COSMICLENS; grant agreement No. 787886) and from the Swiss National Science Foundation (SNSF). This work was supported by World Premier International Research Center Initiative (WPI Initiative), MEXT, Japan. SH acknowledges support by the DFG cluster of excellence "Origin and Structure of the Universe" (www . universe-cluster. de). CDF acknowledges support for this work from the National Science Foundation under Grant No. AST-1715611. SHS thanks the Max Planck Society for support through the Max Planck Research Group. TT acknowledges support by the Packard Foundation through a Packard Research fellowship and by the National Science Foundation through NSF grants AST-1714953 and AST-1906976. LVEK is partly supported through an NWO-VICI grant (project number 639.043.308).

\section{References}

Addison, G. E., Watts, D. J., Bennett, C. L., et al. 2018, ApJ, 853, 119

Alam, S., Ata, M., Bailey, S., et al. 2017, MNRAS, 470, 2617

Arendse, N., Agnello, A., \& Wojtak, R. J. 2019, A\&A, 632, A91

Ata, M., Baumgarten, F., Bautista, J., et al. 2018, MNRAS, 473, 4773

Aylor, K., Joy, M., Knox, L., et al. 2019, ApJ, 874, 4

Bautista, J. E., Vargas-Magaña, M., Dawson, K. S., et al. 2018, ApJ, 863, 110

Bernal, J. L., Verde, L., \& Riess, A. G. 2016, J. Cosmology Astropart. Phys, 10, 019

Blomqvist, M., du Mas des Bourboux, H., \& Busca, N.G. 2019, A\&A, 629, A86

Bonvin, V., Millon, M., Chan, J. H. H., et al. 2019, A\&A, 629, A97

Carneiro, S., de Holanda, P. C., Pigozzo, C., \& Sobreira, F. 2019, Phys. Rev. D, 100, 023505

Carter, P., Beutler, F., Percival, W. J., et al. 2018, MNRAS, 481, 2371

Cattoën, C., \& Visser, M. 2007, CQG, 24, 5985

Chen, G. C. F., Fassnacht, C. D., Suyu, S. H., et al. 2019, MNRAS, 490, 1743

Cuceu, A., Farr, J., Lemos, P., \& Font-Ribera, A. 2019, J. Cosmology Astropart. Phys., 2019, 044

Davis, T. M., Hinton, S. R., Howlett, C., \& Calcino, J. 2019, MNRAS, 490, 2948 de Sainte Agathe, V., Balland, C., \& du Mas des Bourboux, H. 2019, A\&A, 629, A85

Desmond, H., Jain, B., \& Sakstein, J. 2019, Phys. Rev. D, 100, 043537

Doran, M., Stern, S., \& Thommes, E. 2007, J. Cosmology Astropart. Phys., 2007, 015

Eisenstein, D., \& White, M. 2004, Phys. Rev. D, 70, 103523

Foreman-Mackey, D., Hogg, D. W., Lang, D., \& Goodman, J. 2013, PASP, 125, 306

Freedman, W. L., Madore, B. F., Hatt, D., et al. 2019, ApJ, 882, 34

Freedman, W. L., Madore, B. F., Hoyt, T., et al. 2020, ApJ, 891, 57

Gelmini, G.B., Kusenko, A., \& Takhistov, V. 2019, ArXiv e-prints [arXiv:1906.10136]

Goodman, J., \& Weare, J. 2010, Commun. Appl. Math. Comput. Sci., 5, 65

Hannestad, S. 2003, J. Cosmology Astropart. Phys., 2003, 004

Heavens, A., Jimenez, R., \& Verde, L. 2014, Phys. Rev. Lett., 113, 241302

Hu, W., \& Sugiyama, N. 1996, ApJ, 471, 542

Jee, I., Suyu, S. H., Komatsu, E., et al. 2019, Science, 365, 1134

Karwal, T., \& Kamionkowski, M. 2016, Phys. Rev. D, 94, 103523

Kazin, E. A., Koda, J., Blake, C., et al. 2014, MNRAS, 441, 3524

Knox, L., \& Millea, M. 2020, Phys. Rev. D, 101, 043533

Li, E.-K., Du, M., \& Xu, L. 2020, MNRAS, 491, 4960

Li, X., \& Shafieloo, A. 2019, ApJ, 883, L3

Linder, E. V., \& Robbers, G. 2008, J. Cosmology Astropart. Phys., 2008, 004

Millon, M., Galan, A., Courbin, F., et al. 2020, A\&A, in press, https://doi. org/10.1051/0004-6361/201937351

Mörtsell, E., \& Dhawan, S. 2018, J. Cosmology Astropart. Phys., 2018, 025

Planck Collaboration VI. 2020, A\&A, in press https://doi.org/10.1051/ Q004-6361/201833910

Poulin, V., Smith, T. L., Grin, D., Karwal, T., \& Kamionkowski, M. 2018, Phys. Rev. D, 98, 083525

Poulin, V., Smith, T. L., Karwal, T., \& Kamionkowski, M. 2019, Phys. Rev. Lett., 122, 221301

Riess, A. G., Casertano, S., Yuan, W., Macri, L. M., \& Scolnic, D. 2019, ApJ, 876,85

Rigault, M., Aldering, G., Kowalski, M., et al. 2015, ApJ, 802, 20

Risaliti, G., \& Lusso, E. 2019, Nat. Astron., 3, 272

Rusu, C.E., Wong, K.C., \& Bonvin, A. 2019, MNRAS, stz3451

Scolnic, D. M., Jones, D. O., Rest, A., et al. 2018, ApJ, 859, 101

Shajib, A. J., Treu, T., \& Agnello, A. 2018, MNRAS, 473, 210

Sluse, D., Rusu, C. E., Fassnacht, C. D., et al. 2019, MNRAS, 490, 613

Suyu, S. H., Bonvin, V., Courbin, F., et al. 2017, MNRAS, 468, 2590

Taubenberger, S., Suyu, S. H., Komatsu, E., et al. 2019, A\&A, 628, L7

Verde, L., Protopapas, P., \& Jimenez, R. 2013, Phys. Dark Univ., 2, 166

Verde, L., Bernal, J. L., Heavens, A. F., \& Jimenez, R. 2017, MNRAS, 467, 731

Visser, M. 2004, CQG, 21, 2603

Weinberg, S. 1972, Gravitation and Cosmology: Principles and Applications of the General Theory of Relativity (Wiley-VCH)

Wojtak, R., \& Agnello, A. 2019, MNRAS, 486, 5046

Wong, K.C., Suyu, S.H., Chen, G.C.F., et al. 2020, MNRAS, in press [arXiv:1907.04869]

Yang, T., Banerjee, A., \& Colgáin, E. 2019, ArXiv e-prints [arXiv:1911.01681]

Yıldırım, A., Suyu, S. H., \& Halkola, A. 2020, MNRAS, 493, 4783

Yuan, W., Riess, A. G., Macri, L. M., Casertano, S., \& Scolnic, D. M. 2019, ApJ, 886,61 


\section{Appendix A: Planck compressed likelihood}

Much of the constraining power of the CMB power spectrum can be compressed in three parameters: the physical density of baryons $\Omega_{\mathrm{b}} h^{2}$, which determines relative heights of the peaks in the power spectrum, and two so-called shift parameters that describe two fundamental and directly measured angular scales related to the sound horizon and the Hubble horizon at the time of decoupling. The shift parameters are defined by the following equations:

$\mathcal{R}=\sqrt{\Omega_{\mathrm{m}}} \frac{D_{\mathrm{A}}\left(z_{*}\right)}{H_{0}^{-1}}$,

$\theta_{*}=\frac{r_{\mathrm{s}}\left(z_{*}\right)}{D_{\mathrm{A}}\left(z_{*}\right)}$,

where $z_{*}$ is redshift of decoupling and $D_{\mathrm{A}}$ is the comoving angular diameter distance, which for flat models considered in this work is given by

$D_{\mathrm{A}}=\mathrm{c} \int_{0}^{z} \frac{\mathrm{d} z}{H(z)}$

$H^{2}(z)=H_{0}^{2}\left[\Omega_{\mathrm{m}}(1+z)^{3}+\Omega_{\mathrm{DE}}(z)+\Omega_{\gamma}(1+z)^{4}\right]$,

where $\Omega_{\gamma}$ denotes the density parameter of radiation, meaning $\Omega_{\gamma}=2.47 \times 10^{-5} h^{-2}$.

The comoving sound horizon is given by

$r_{\mathrm{s}}(z)=\frac{\mathrm{c}}{\sqrt{3}} \int_{z}^{\infty} \frac{\mathrm{d} z}{H(z) \sqrt{1+\frac{3 \Omega_{\mathrm{b}}}{4 \Omega_{\gamma}}(1+z)^{-1}}}$.

Here, an additional contribution to the energy density driving the expansion comes from relativistic neutrinos. The density parameter of relativistic neutrinos $\Omega_{\mathrm{n}}$ is given by

$\Omega_{\mathrm{n}}=N_{\mathrm{eff}} \frac{7}{8}\left(\frac{4}{11}\right)^{4 / 3} \Omega_{\gamma}$,

where $N_{\text {eff }}$ is the effective number of neutrinos, with $N_{\text {eff }}=3.046$ for the baseline model.

We computed redshift $z_{*}$ of decoupling employing the following fitting formula (Hu \& Sugiyama 1996):

$$
\begin{aligned}
z_{*} & =1047\left[1+0.00124\left(\Omega_{\mathrm{b}} h^{2}\right)^{-0.738}\right]\left[1+g_{1}\left(\Omega_{\mathrm{m}} h^{2}\right)^{g_{2}}\right] \\
g_{1} & =0.0783\left(\Omega_{\mathrm{b}} h^{2}\right)^{-0.238}\left[1+39.5\left(\Omega_{\mathrm{b}} h^{2}\right)^{0.763}\right]^{-1} \\
g_{2} & =0.56\left[1+21.1\left(\Omega_{\mathrm{b}} h^{2}\right)^{1.81}\right] .
\end{aligned}
$$

The sound horizon imprinted in galaxy clustering and measured from BAO observations is fixed at the drag epoch, when the baryons are released from the Compton drag of the photons. The corresponding drag redshift $z_{\mathrm{d}}$ can be calculated using the following fitting function (Hu \& Sugiyama 1996):

$$
\begin{aligned}
& z_{\mathrm{d}}=1345 \frac{\left.\left(\Omega_{\mathrm{m}} h^{2}\right)^{0.251}\left[1+b_{1}\left(\Omega_{\mathrm{b}} h^{2}\right)^{b_{2}}\right)\right]}{1+0.659\left(\Omega_{\mathrm{m}} h^{2}\right)^{0.828}} \\
& b_{1}=0.313\left(\Omega_{\mathrm{m}} h^{2}\right)^{-0.419}\left[1+0.607\left(\Omega_{\mathrm{m}} h^{2}\right)^{0.674}\right] \\
& b_{2}=0.238\left(\Omega_{\mathrm{m}} h^{2}\right)^{0.223}
\end{aligned}
$$

The compressed CMB likelihood is given by a threedimensional Gaussian distribution in the three parameters mentioned above, meaning $\Omega_{\mathrm{n}} h^{2}, \mathcal{R}$, and $\theta_{*}$. We employed the mean values and the covariance matrix determined from publicly available MCMC models obtained for a flat $\Lambda$ CDM

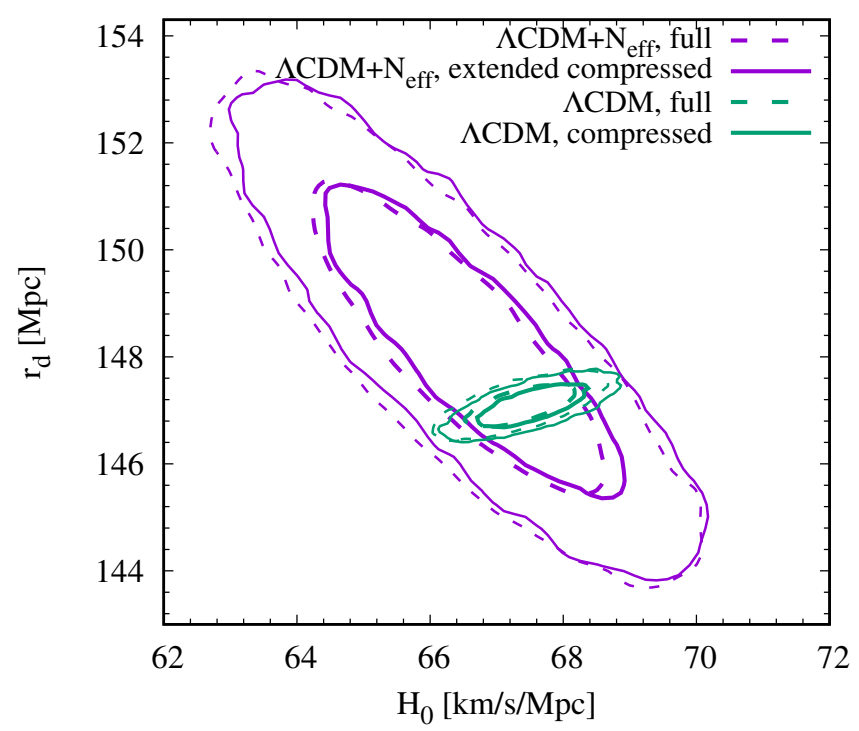

Fig. A.1. Comparison between constraints on $r_{\mathrm{d}}$ and $H_{0}$ from the full Planck likelihood (dashed lines) and the compressed likelihood (for post-recombination modifications of $\Lambda \mathrm{CDM}$ ) or the extended compressed likelihood (for pre-recombination modifications of $\Lambda \mathrm{CDM}$ ) used in this study (solid lines). The robustness test comprises two cases: the standard flat $\Lambda \mathrm{CDM}$ model and its extension with a free number of neutrinos.

model fitted to the Planck observations, including the temperature, polarisation, and lensing data (Planck Collaboration VI 2020): $\left(100 \Omega_{\mathrm{b}} h^{2}, 100 \theta_{*}, \mathcal{R}\right)=(2.237 \pm 0.015,1.0411 \pm$ $0.00031,1.74998 \pm 0.004)$ with the following correlation matrix:

$$
\left(\begin{array}{ccc}
1.00 & 0.34 & -0.63 \\
0.34 & 1.00 & -0.46 \\
-0.63 & -0.46 & 1.00
\end{array}\right)
$$

The compressed likelihood accurately recovers the actual constraints obtained from the complete likelihood for a flat $\Lambda C D M$ model (see Fig. A.1). Only a fine adjustment of the redshift scales in both fitting formulae $\left(\delta z / z \sim 10^{-3}\right.$, smaller relative to the values adopted in Hu \& Sugiyama 1996) was applied in order to correct for a sub-percent bias in the mean values of relevant parameters. In general, both approximations used to compute $z_{*}$ and $z_{\text {drag }}$ are accurate to within 1 per cent in a wide range of the matter and baryon density parameters ( $\mathrm{Hu} \&$ Sugiyama 1996).

For early-time extensions of the standard $\Lambda \mathrm{CDM}$ cosmology (such as a model with free $N_{\text {eff }}$ ), the compressed likelihood turns out to be insufficient, leading to a family of models with a wide range of amplitudes of the first peak in the power spectrum. In order to circumvent this problem, we extended the compressed likelihood described above by accounting for the height of the first peak in the power spectrum as an additional constraint. Bearing in mind that the amplitude scales with $\Omega_{\mathrm{dm}} h^{2}$, meaning the physical density of dark matter, a simple extension relies on adding $\Omega_{\mathrm{dm}} h^{2}$ as the fourth variable in the compressed likelihood function. Using Planck results for a $\Lambda \mathrm{CDM}$ model with a free effective number of neutrinos as a base earlytime extension (inferred from the full temperature and polarisation data), we determined the mean values and the covariance matrix of the new four-parameter compressed likelihood, obtaining $\left(100 \Omega_{\mathrm{b}} h^{2}, 100 \theta_{*}, \mathcal{R}, \Omega_{\mathrm{dm}} h^{2}\right)=(2.225 \pm 0.0223,1.0414 \pm$ $0.00054,1.7529 \pm 0.0056,0.1184 \pm 0.0029)$ and the following 
correlation matrix:

$\left(\begin{array}{cccc}1.00 & -0.50 & -0.79 & 0.51 \\ -0.50 & 1.00 & 0.30 & -0.81 \\ -0.79 & 0.30 & 1.00 & -0.19 \\ 0.51 & -0.81 & -0.19 & 1.00\end{array}\right)$.

Figure A.1 demonstrates that the extended compressed likelihood accurately recovers the actual constraints on $r_{\mathrm{d}}$ and $H_{0}$ from Planck for a model with a free effective number of neutrinos.

\section{Appendix B: Polynomial parametrisations}

This section gives more detailed information about the polynomial parametrisations used throughout this work.

\section{B.1. Expansion formulae}

Our first model is the simplest one and adopts a polynomial expansion of $H(z)$ in $z$;

$H(z)=H_{0}\left[1+b_{1} z+b_{2} z^{2}+O\left(z^{3}\right)\right]$,

where $H_{0}$ is the Hubble constant, and the coefficient $b_{1}$ is related to the deceleration parameter $q_{0}$ through

$b_{1}=1+q_{0}$.

In our second model, the luminosity distance $D_{L}$ is expanded as a polynomial in $\log (1+z)^{7}$;

$x=\log (1+z)$,

$D_{L}(z)=\frac{c \ln (10)}{H_{0}}\left[x+c_{2} x^{2}+c_{3} x^{3}+c_{4} x^{4}+O\left(x^{5}\right)\right]$,

where the coefficient $c_{2}$ is related to the deceleration parameter through the following relation:

$c_{2}=\frac{\ln (10)}{2}\left(2-q_{0}\right)$

This different parametrisation was chosen in order to avoid convergence problems with the Taylor expansion around zero, when employing data with redshifts $z>1$. By introducing a new variable $x$ that satisfies $x=0$ when $z=0$, and $x<1$ when $z \rightarrow 2$ (where the upper limit of 2 is based on the highest lensed quasar redshift), the parametrisation is kept within the convergence radius of the Taylor expansion.

Our third model describes transverse comoving distances $D_{M}$ by polynomials in $z /(1+z)$;

$y=\frac{z}{1+z}$,

$D_{M}(z)=\frac{c}{H_{0}}\left[y+d_{2} y^{2}+d_{3} y^{3}+d_{4} y^{4}+O\left(y^{5}\right)\right]$,

where the coefficient $d_{2}$ is related to the deceleration parameter through

$d_{2}=\frac{1}{2}\left(1-q_{0}\right)$.

This parametrisation was, similarly to the one in model 2 , chosen to overcome convergence problems.
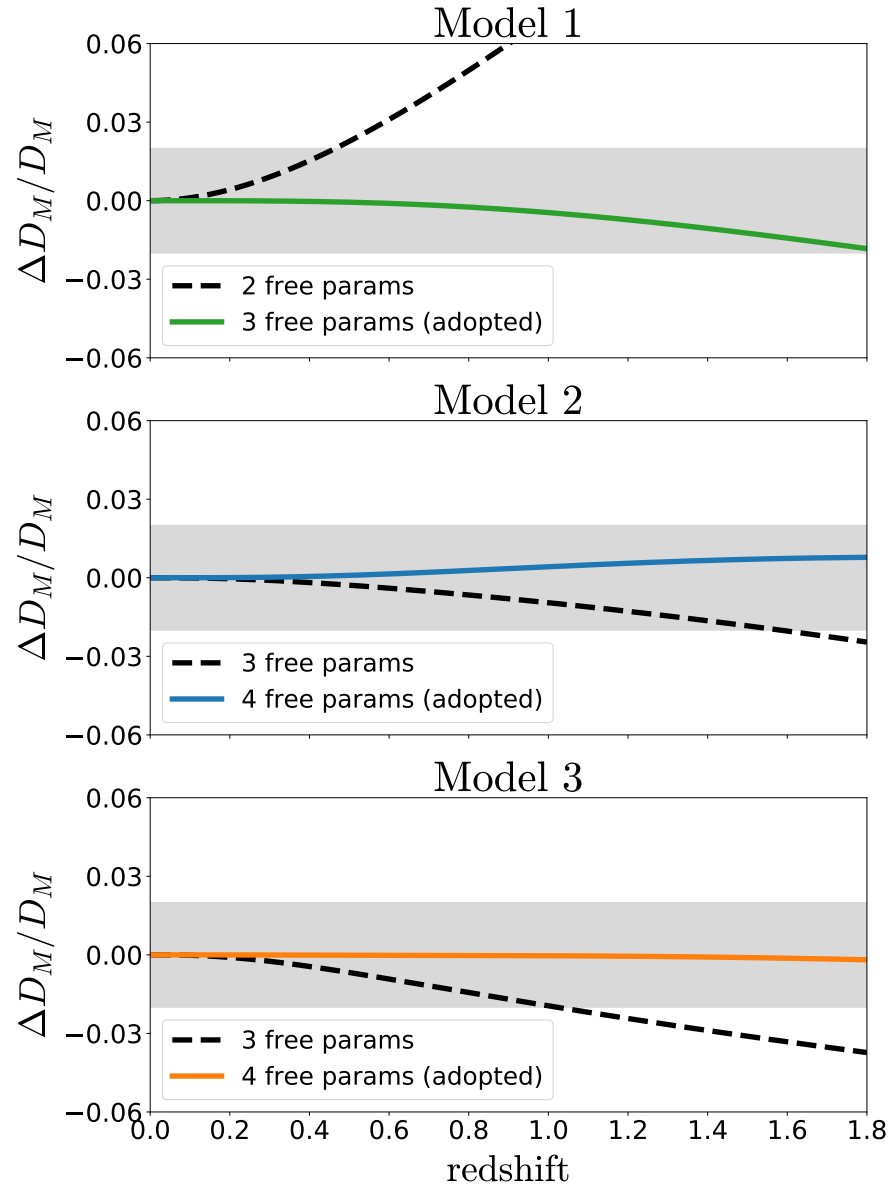

Fig. B.1. Relative differences between distances in a fiducial flat $\Lambda \mathrm{CDM}$ model and distances derived from models 1-3 with free parameters matched to the kinematical coefficients of the fiducial model, $\Delta D_{\mathrm{M}} / D_{\mathrm{M}}=\left(D_{\mathrm{M}, \text { expansion }}-D_{\mathrm{M}, \Lambda \mathrm{CDM}}\right) / D_{\mathrm{M}, \Lambda \mathrm{CDM}}$. The solid lines show the results satisfying the convergence criterion, which sets the truncation of polynomials used in the adopted models in this study.

\section{B.2. Truncation of the polynomials}

An important thing to consider is at which order the Taylor expansions should be truncated. Higher orders of expansions can give better approximations to the shape of the data, but also introduce more free parameters and therefore larger uncertainties. In order to determine the truncation of the polynomials as given in Eqs. (B.1), (B.3), and (B.5), we performed a convergence test to check that the models can accurately recover expansion history of a fiducial flat $\Lambda C D M$ cosmological model in a redshift range of observational data used in our study, for instance, $z<1.8$. The test relies on comparing distances from models $1-3$ to the actual distances in the fiducial model. Free parameters of the models were determined by matching coefficients of Taylor expanded Hubble parameter in models 1-3 and the fiducial model. The latter yields well-known kinematical coefficients (Weinberg 1972; Visser 2004):

$q_{0}=\frac{3}{2} \Omega_{m}-1$,

$j_{0}=1$,

$s_{0}=1-\frac{9}{2} \Omega_{m}$.

7 Here, $\log (1+z)$ refers to the $\log$ base 10 , and not to the natural logarithm. 

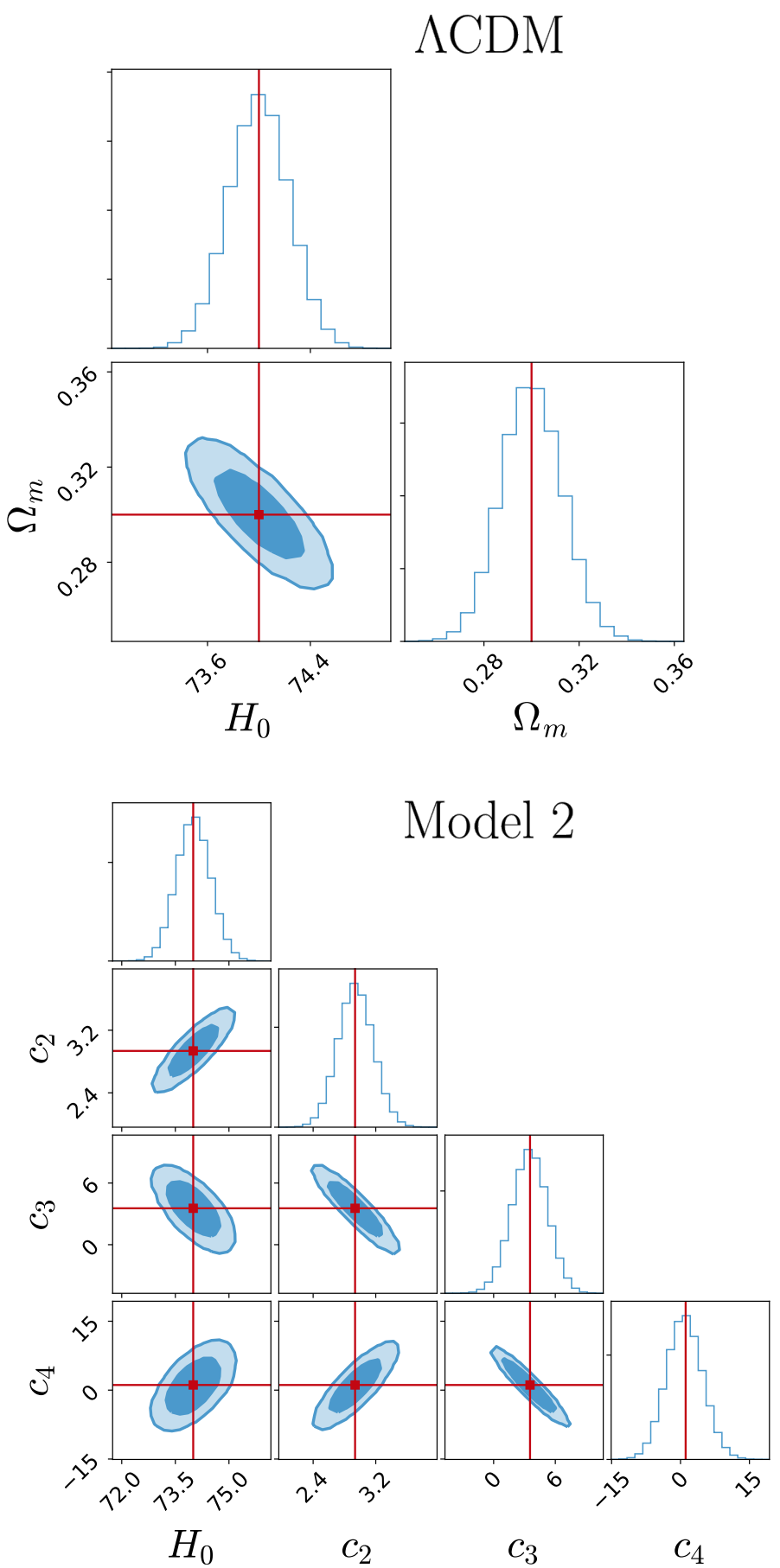
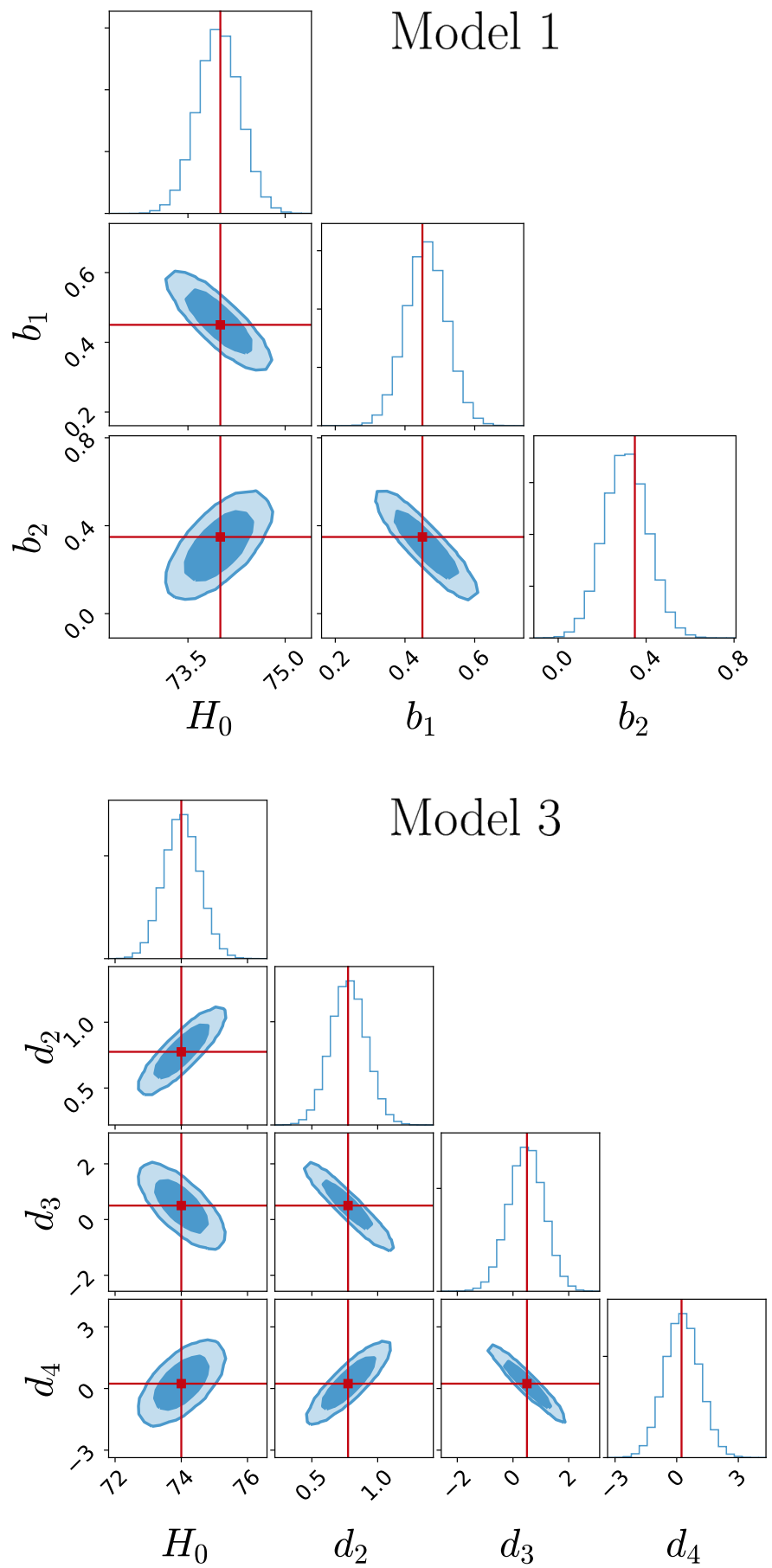

Fig. B.2. Best-fit values of flat $\Lambda \mathrm{CDM}$ and polynomial parametrisation models 1-3 to mock data. The mock data is generated by replacing the Pantheon distance modulus points by their fiducial flat $\Lambda \mathrm{CDM}$ values. The red lines indicate the canonical $\Lambda \mathrm{CDM}$ values of $\Omega_{\mathrm{m}}, H_{0}$ and the expansion coefficients $b_{i}, c_{i}$, and $d_{i}$.

Since the errors that we obtain by combining calibrations of HOLiCOW and SHOES are around $2 \%$ (see Table 3), we required our models to be within a $2 \%$ accuracy of $\Lambda$ CDM distances in this test. The results can be seen in Fig. B. 1 for $\Omega_{\mathrm{m}}=0.3$, where the shaded region corresponds to this imposed limit. It is sufficient to employ three free parameters (corresponding to a second-order polynomial) for model 1 and four free parameters (corresponding to a fourth-order polynomial) for models 2 and 3 to satisfy the convergence condition. Since a further increase of the number of free parameters is disfavoured by the BIC obtained in fits with the actual late-time observations, these polynomial truncations were adopted in our study (see Table 1). The BIC score is calculated as

$\mathrm{BIC}=\ln (N) k-2 \ln \left(\mathcal{L}_{\text {m.a.p. }}\right)$,

where $N$ is the number of data points and $k$ is the number of all free parameters in the cosmological fits. 


\section{B.3. Test with mock distance-modulus data}

As a final test for our polynomial parametrisation models, we investigated if any biases were introduced when we fitted models 1-3 to flat $\Lambda$ CDM data. We transformed the Pantheon SN data set to a mock data set by replacing their binned distance modulus entries with the fiducial flat $\Lambda \mathrm{CDM}$ values (adopting $H_{0}=74 \mathrm{~km} \mathrm{~s}^{-1} \mathrm{Mpc}^{-1}$ and $\Omega_{\mathrm{m}}=0.3$ ) at the same redshifts. For the errors associated with the distance moduli we keep the original Pantheon ones. By construction, best fit $\Lambda$ CDM parameters are equal to their fiducial values, whereas relative shifts in best fit parameters obtained for non- $\Lambda \mathrm{CDM}$ models measure the corresponding biases. This test is similar to the one performed by Yang et al. (2019), in which they found that our model 2 introduces an artificial bias. However, their mock data set is based on Pantheon data as well as high-redshift quasar and GRB data (with $z_{\max }=6.7$ ), while in our work we only used sources below $z=1.8$. Figure B.2 shows the best fit values for the coefficients $b_{i}, c_{i}$, and $d_{i}$ of models 1-3, obtained with MCMC, and their true values in a flat $\Lambda \mathrm{CDM}$ cosmology. As can be seen, they are in complete agreement with each other. In fact, the relative difference in $H_{0}$ between the fiducial value and those of models
$1-3$ is $0.03 \%, 0.02 \%$, and $0.02 \%$, respectively. This bias is about a hundred times smaller than the current precision achieved by SHOES and HOLiCOW data (which is around $2 \%$ ). The bias in $q 0$ is larger: $2.0 \%, 1.2 \%$, and $1.3 \%$ for models $1-3$, but still negligible compared to our obtained errors in $q_{0}$ (which are $10 \%$ at best).

This test demonstrates that if the underlying cosmology is flat $\Lambda \mathrm{CDM}$, then our models will not introduce any significant biases in the Pantheon redshift range. The convergence test in the previous section also guarantees this. The bias that Yang et al. (2019) found in their model was a consequence of it not passing the convergence test over the complete redshift range of $z=0-7$.

We repeated the test for the PEDE model and for a $w C D M$ cosmology with $w=-1.2$. In both cases, we assumed $\Omega_{\mathrm{m}}=0.3$. We found only a sub-percent bias in the best fit $H_{0}$ and a fewpercent bias in $q_{0}$, where the actual values are given by;

$q_{0, \mathrm{PEDE}}=\frac{3}{2} \Omega_{\mathrm{m}}-\frac{1-\Omega_{\mathrm{m}}}{2 \ln (10)}-1$,

$q_{0, w \mathrm{CDM}}=\frac{1}{2}+\frac{3}{2} w\left(1-\Omega_{\mathrm{m}}\right)$. 